\title{
Bayesian modeling of pilot belief and visual misperception in helicopter overland navigation
}

\author{
Ji Hyun Yang and Quinn Kennedy \\ Operations Research Department and MOVES Institute \\ Naval Postgraduate School \\ Monterey, CA \\ jyan1@nps.edu; mqkenned@nps.edu
}

\author{
Joseph Sullivan and Michael Day \\ MOVES Institute \\ Naval Postgraduate School \\ Monterey, CA \\ jasullivan@nps.edu; maday@nps.edu
}

\begin{abstract}
This paper aims to provide a framework to model human belief and misperception in helicopter overland navigation. Helicopter overland navigation is known to be a challenging cognitive task, and understanding the cognitive processes associated with it is non-trivial. Twelve military personnel participated in the study and statistical analysis showed that their gaze parameters can be predicted by their level of expertise. Some pilots showed common visual misperception during the navigation task, which can be explained by the following errors: 1) confusion between inference and evidence, 2) incorrect mutually exclusive assumptions on the data, and 3) biased sampling. Simulation results on two cases observed in the experiments are given. Quantitative differences in dynamic perceptions between a Bayesian agent and misperceiving humans are presented with the suggested modeling framework.
\end{abstract}

Keywords - mispercetion; visual perception; Bayesian modeling; cognition; navigation

\section{INTRODUCTION}

In many different disciplines such as psychology, neuroscience, and cognitive engineering, research efforts have been focused on understanding human perception. For example, Brunswikian theory and method (the lens model) has been employed to the technological mediation of perception in many substantive domains including aviation, military applications, and dynamic system, and human-computer interaction [1]. Bayesian inference has also been widely used to model many different aspects of cognition, such as induction bias [2, 3]. Reference [2] showed that a Bayesian framework can model biases captured through empirical data.

Helicopter overland navigation is a complex task that requires continuous visual cue perception and decision making and is normally assigned to a non-flying pilot. However, how the non-flying pilot perceives visual cues in the OTW (out-thewindow) view and how they make a series of navigational decisions based on their perceptions is not well understood. Most human cognitive modeling techniques assume that humans are as reasonable as in a Bayesian framework, i.e., following Bayes' rule: maximizing or minimizing certain criteria to update prior knowledge. Such approaches generally do not include human errors such as misperception and confusion. Although uncertainty parameters in the models can be set to capture misperception, it would be very useful to understand and model such errors specifically rather than lump them into uncertainties. For example, what are common

This work is sponsored by U.S. Navy Modeling Simulation Office (NMSO) and Office of Naval Research (ONR). misperceptions, when are these errors made, and how can they be modeled quantitatively? Currently, observing a pilot's cognitive state is guesswork and there are few cues available to assess it. If we can monitor when and how misperceptions or mistakes are made, this information would have an important impact on training effectiveness of student pilots. Feeding misperception information in real time to the instructor pilot would help both the instructor to know what is going on and the student pilot to realize what is wrong in a specific way.

Coherence theory [4] illustrates the relationship between visual perception, cognition, and human error. It posits that in terms of scene representation, attention shapes a dynamic representation of the scene that is strongly influenced by task demands and observer expectations. For example, coherence theory can explain visual misperception phenomena of change blindness and inattentional blindness. Change blindness occurs when people do not detect changes in scenes that were made during blinks, flickers, or disruptions [5]. Inattentional blindness [6] occurs when people are overly focused on certain aspects of the scene or on a visual task such that that they do not see obvious changes, e.g., "Gorillas in our Midst experiment" by Simons \& Chabris [7]. In the Gorillas in our Midst experiment, participants tasked with counting the number of passes made by one basketball team during a game did not see a person in a gorilla suit walk to the middle of the court, beat their chest, and walk off the court. When these visual misperceptions are coupled with an overconfidence bias [8], decisions are affected.

Research on human error detection modeling [e.g., 9] could help understand some types of human error. Some approaches [e.g., 10] have tried to integrate different cognitive functions. However, visual misperception has not been specifically studied in overland navigation. Thus, we provided a formal framework to model human misperception in helicopter overland navigation task as our representative cognitive task. We monitored pilots' visual scan patterns, tracked their OTW view and navigation performance. The navigation task is described in Section II along with a summary of statistical analyses on pilots' visual scan patterns. Section III explains in detail the observed visual misperception during the navigation task and provides a formal model technique of the misperception using a Bayesian framework. Various simulation results comparing reasonable Bayesian agents and errormaking humans in two different cases observed in the 
experiments are shown in Section IV. Section V summarizes the study and provides future research ideas and plans.

\section{OVERLAND NAVIGATION AND EMPIRICAL OBSERVATION ON VISUAL PERCEPTION}

The navigation task was to fly over 12 waypoints (indicated as black circles on Fig. 1) in a simulated terrain model of Twentynine Palms, CA after studying the area utilizing the Falcon View flight planning software system. The first waypoint is located slightly south of the map so it is not shown in Fig. 1. Each waypoint pair has a "doghouse" that indicates (from top to bottom): the next waypoint number, the recommended heading to reach that waypoint from the previous one, the distance between waypoints, and the amount of time it takes to traverse the distance assuming a speed of about 60 knots. The task was created to be challenging. Waypoints are very close together and the terrain tends to be ambiguous, so subjects needed to make course corrections based on visual cues from both the OTW and map screens (their goal being to bring their perceived location closer to their actual location). Details of the experiments including laboratory setting can be found in [11-13].

Twelve male military personnel, 29 to 40 years of age participated in the study. Total flight hours (TFH) ranged from $0-2700$. Minimum skill requirement for the study was completion of at least one overland navigation class. Expertise effect on the flight performance such as deviation from the flight trajectory and visual scan parameters such as dwell duration can be found in [11]. In this paper, our focus is on the modeling of visual perception/misperception, i.e., how pilots match visual cues from OTW to terrain features on the map while navigating based on the navigation trajectory and the corresponding OTW view we collected.

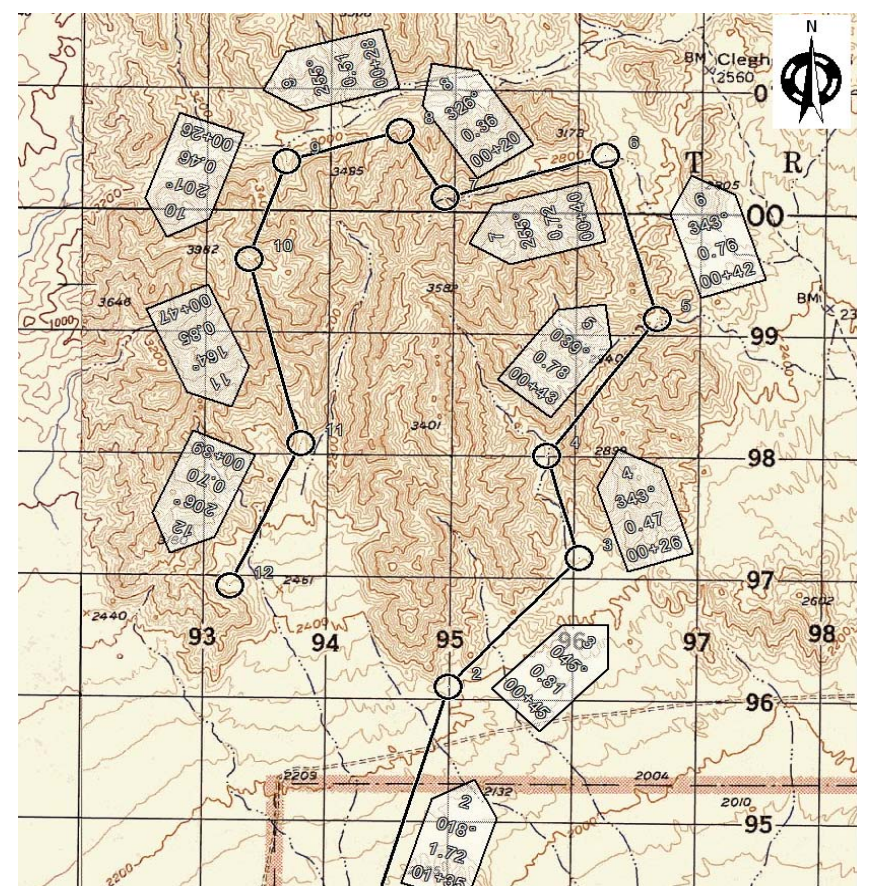

Figure 1. Main flight route showing $2^{\text {nd }}-12^{\text {th }}$ waypoints with corresponding doghouses [5] at Twentynine Palms, CA
One of the typical navigation problems that pilots confront can be described as follows. Suppose pilots see the OTW view as shown in Fig. 2(a), i.e., hills on both the left and right making a valley in view. This type of valley is a very common feature in the terrain and a challenge of pilots is to locate themselves in the map (Fig.1) based on the OTW terrain feature along with their previous cues. For example, markers A and B in Fig. 2(b) are candidate locations corresponding to the OTW view of Fig. 2(a). The OTW cue itself is incomplete for pilots to decide which valley they are facing. Thus, pilots use their previous knowledge or beliefs to decide whether they are located either on A or B.

What we have observed through the experiment suggest that pilots (especially with less experience) tend to perceive these OTW cues in a biased way, favoring their prior beliefs. Instead of making a fair estimate, some pilots seemed to choose the location they need to navigate rather than considering other possible locations. Some believed what they expected instead of what they saw. Some only sampled visual cues in view which are compatible with their current belief and disregarded cues that do not fit with their current belief. These visual perception patterns were observed through the whole navigation experiment, and we decided to model those specific visual misperceptions. Section III describes, categorizes and models visual misperceptions in Bayesian framework.

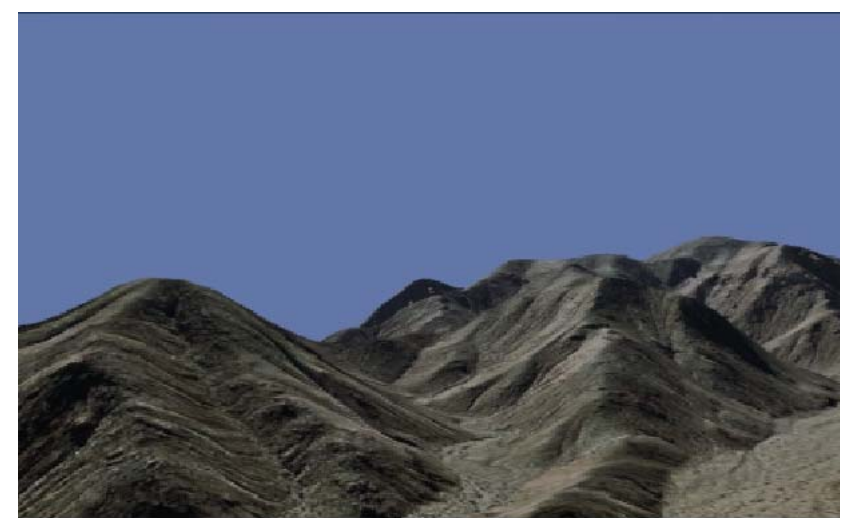

(a)

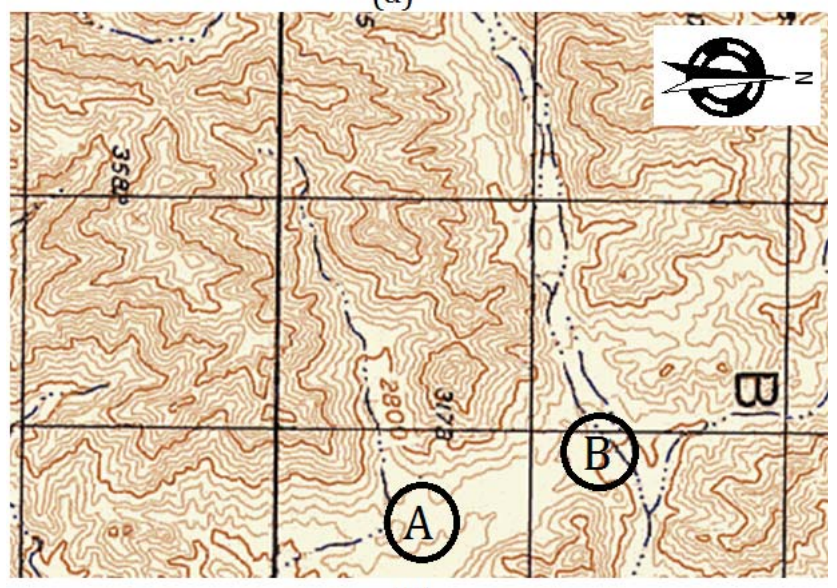

(b)

Figure 2. (a) typical OTW view with hills/valleys and (b) candidate locations of the OTW view 


\section{BAYESIAN MODELING OF VISUAL MISPERCEPTION IN A HELICOPTER OVERLAND NAVIGATION TASK}

Some expert pilots successfully located waypoint six and made a 90 degree left turn into a narrow valley toward waypoint seven. However, nine out of twelve pilots missed this narrow valley mainly due to a field of view angle limitation. Once they passed waypoint six without realizing it, another valley appeared on their left. Pilots who missed waypoint six made a left turn into this valley believing they were on track.

As shown in Fig. 3, subject 5 missed waypoint 6 and took a left turn into this valley $\left(6^{\prime}\right)$ as shown in Fig. 3. Then, he flew north of the intended trajectory ( $7^{\prime}$ and $\left.8^{\prime}\right)$, believing he was on waypoints 7 and 8 . Initially planned waypoints are shown in black whereas the subject's estimation is shown in blue. On his way from waypoint 6 ' to 7 ', he saw a valley on the right side of the flight heading direction in the OTW scene. If he had been on track (i.e., between 6 and 7), he would have been surrounded by hills and should not have been able to see any saddle or valley and his heading would have been much different. Even though his gaze data showed that he scanned the valley, the pilot did not question his orientation. This indicates the pilot rejected the visual cues that were not compatible with his current belief, which could not have been correct. Thus, the subject did not question his orientation or status, indicating that he overweighed those visual cues that fit into his hypothesis by giving little attention (subconsciously) to cues conflicting with it. This type of bias, carrying over initial bias, has also been seen in a cognitive task that tapped inductive biases on cultural evolution [2].

The visual misperception explained above can be understood in Bayesian framework. Suppose a navigating pilot updates his belief and/or confidence on his position whenever he sees salient terrain features, i.e., visual cues. The terrain features in the pilot's sights are information $(d)$ provided to the pilot, who infers his/her current position $(H)$ based on the data. Thus, the probability of a pilot's current position being at waypoint six after seeing a valley (i.e., hills on the left and right) can be obtained by applying Bayes' rule.

$$
p(H \mid d)=\frac{p(d \mid H) \cdot p(H)}{p(d \mid H) \cdot p(H)+p(d \mid \sim H) \cdot p(\sim H)}
$$

where $p(H)$ is the pilot's belief probability before seeing the scene $d$, (i.e., hills on the left and right), $p(d \mid H)$ is the conditional probability that the pilot sees the scene when pilot is at waypoint six, and $p(d \mid \sim H)$ is the conditional probability that the pilot sees the same scene when the pilot is in the other valley. For simplicity, we only consider two possible hypotheses: locations 6 and 6 , i.e., $H$ and $\sim H$ respectively, as described in the previous paragraph. Then, we have $p(\sim H)=$ $l \quad p(H)$. Both valleys have hills on the left and right, and the pilot is equally likely to see the terrain features when the pilot is at waypoint six $(H)$ or in the other valley $(\sim H)$. Then,

$$
p(d \mid H)=p(d \mid \sim H)
$$

If a pilot realizes this fact, his posterior belief $p(H \mid d)$ should be unchanged from his prior belief $p(H)$. An elementary calculation yields $p(H \mid d)=p(H)$ from Bayes' rule. However, our experiments showed that the initial bias not only carried on as shown in [2], but also amplified favoring the initial bias.

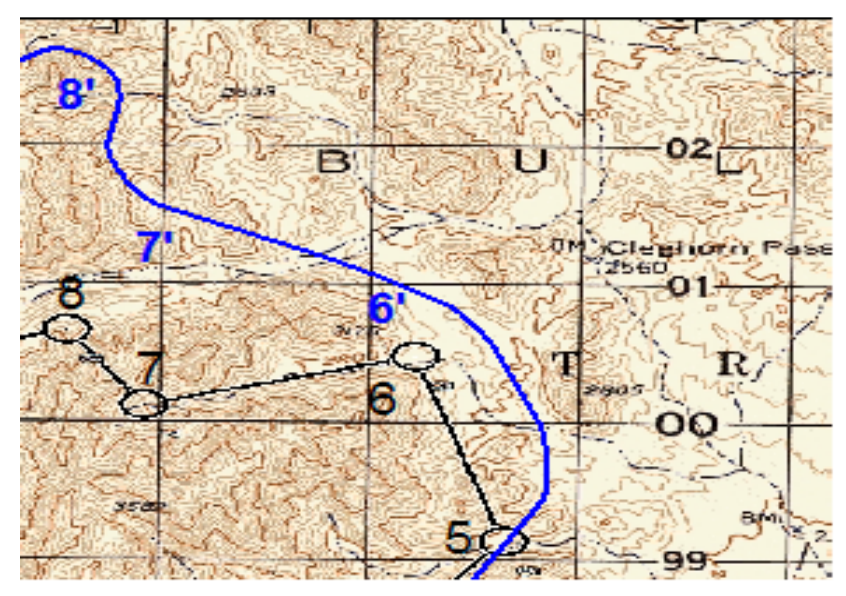

Figure 3. Subject 5's actutal flight trajectory (blue) and planned route (black)

This bias was especially obvious for less experienced pilots; they seemed to believe what they expected to see instead of what they actually saw. This misperception can be explained by three human errors. First, pilots experienced confusion between inference $p(H \mid d)$ and evidence $p(d \mid H)$ especially when $p(d \mid H)$ is high. Because pilots already believed that they were on-track and the scene was a very likely cue, i.e., $p(d \mid H) \approx 1$, they approximated $p(H \mid d) \approx p(d \mid H) \approx 1$, which is not a correct estimation. These pilots chose an easy, inaccurate approximation instead of inferring in a non-biased manner. This confusion pushed the initial bias the wrong way.

Second, pilots could incorrectly assume mutually exclusive events from the evidence, i.e., $p(d \mid \sim H)=1-p(d \mid H)$ as opposed to the correct assumption shown in Eq. (2) $p(d \mid H)=p(d \mid \sim H) \approx$ 1. The terrain feature they see is neither a unique nor an exclusive visual cue, rather it has multiple solutions. However, pilots sometimes overweighed the visual cue favoring their initial bias: they did not consider the possibility of the visual cue being from another valley $(\sim H)$.

Third, pilots sampled data in a biased manner as in inattentional blindness [6]. They disregarded cues which were not compatible with their current belief. They did not update their belief when the OTW view included visual cues incompatible with their current hypothesis. However, when they were given a compatible cue, they used the cue to solidify their possibly wrong hypothesis as shown in the two previous misperception types. Table I summarizes visual misperception modeling introduced in this section.

TABLE I. VISUAL PERCEPTION AND MISPERCEPTION MODELING

\begin{tabular}{|c|l|}
\hline $\begin{array}{c}\text { Perception } \\
\text { type }\end{array}$ & Posterior probability \\
\hline $\begin{array}{c}\text { Bayesian agent } \\
\text { (A) }\end{array}$ & $p(H \mid d)=\frac{p(d \mid H) \cdot p(H)}{p(d \mid H) \cdot p(H)+p(d \mid \sim H) \cdot p(\sim H)}$ \\
\hline $\begin{array}{c}\text { Misperception } \\
\text { Type 1 (B1) }\end{array}$ & $p(H \mid d)=p(d \mid H) \quad$ when $p(d \mid H) \approx 1$ \\
\hline $\begin{array}{c}\text { Misperception } \\
\text { Type 2 (B2) }\end{array}$ & $p(H \mid d)=\frac{p(d \mid H) \cdot p(H)}{p(d \mid H) \cdot p(H)+(1-p(d \mid H)) \cdot p(\sim H)}$ \\
\hline $\begin{array}{c}\text { Misperception } \\
\text { Type 3 (B3) }\end{array}$ & $p(H \mid d)=p(H) \quad$ when $p(d \mid H) \approx 1$ \\
\hline
\end{tabular}




\section{DECISION BIAS SIMULATION: BAYESIAN AGENT VS. MISPERCEIVING HUMAN}

Based on experimental observation, misperception modeling in a Bayesian framework was introduced in the previous section. Simulation results with the modeling framework are given in this section. Our objective is to quantify human misperception evolving over time and to simulate the gap between a series of "misperceptions" and "correct decisions." A correct decision is assumed to adapt Bayes' rule with non-biased samplings of data. We use the following notation in this section: $\mathrm{A}=$ always correct agent, i.e., a Bayesian agent who always adapts Bayes' Rule to update its inferential probability, $\mathrm{B}=$ misperceiving human, i.e., an agent who uses misperception models introduced in Table I to update its inferential probability.

\section{A. Case 1: single point inference update}

Let's first examine the case introduced in the beginning of Section III: Nine out of twelve pilots missed waypoint 6 due to a view angle limitation. Once they passed waypoint 6 without realizing it, another valley appeared on their left. Pilots who missed waypoint six made left turns into this valley believing they were on-track.

We can assume the prior inference $p(H)$ at waypoint 5 was high (for example, .9) because the subject's actual position as well as perception was rational and on-track. Then, the OTW visual cue including hills on the left and right appeared which is very likely to be a cue for both $H$ and $\sim H$., i.e., $p(d \mid H)=$ $p(d \mid \sim H)=.95$. As stated in Section III, posterior probability does not change from prior when applying Bayes' rule. Thus, agent A's $p(H)$ remains the same even after seeing the visual cue. However, misperception occurs in agent $\mathrm{B}$, which results in increasing $p(H)$. Fig. 4(a) shows prior and posterior inferential probabilities of agents A and Bs respectively. Agent A's probability does not change after seeing the visual cue, whereas agent B1 and B2's probability increased according to the misperception type they adapted.

Fig. 4(b) compares biased sampling and Bayesian perception when given a very unlikely cue for $H$ but not so much for $\sim H$, i.e., $p(d \mid H)=.1$ and $p(d \mid H)=.5$. Agent B3 disregards the cue which is not compatible with the prior and keeps the prior as the posterior inferential probability. However, agent A applied Bayes' rule and updated its inference from .9 to .643 .
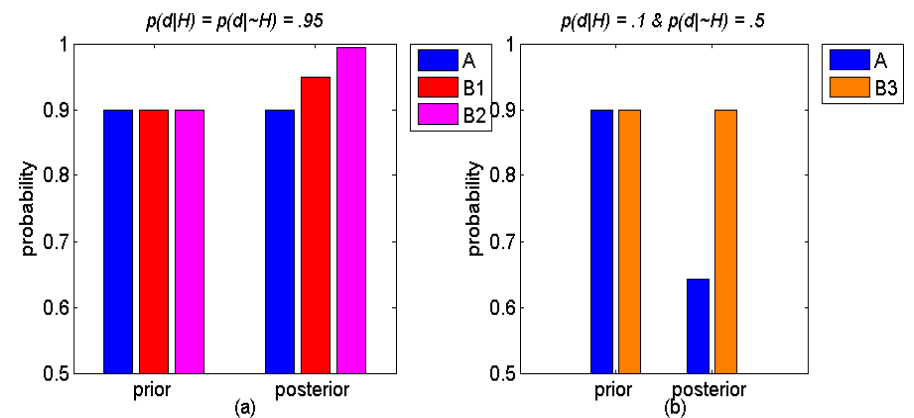

Figure 4. Differences in posterior inferential probabilities given same priors A: Bayesian agent, B1: Misperception type 1, B2: Misperception type 2, B3: Misperception type 3 .
Fig. 4(a) shows a case when the prior $p(H)$ and $p(d \mid H)=$ $p(d \mid \sim H)$ were set to fixed values, i.e., .9 and .95 . Fig. 5 describes differences between agent A, B1 and B2's inferential probabilities when the prior $p(H)$ changes from 0 to 1 . The visual cue used in this simulation was a cue which is highly plausible and non-unique, i.e., $p(d \mid H)=p(d \mid \sim H)=.95$. The OTW visual cues shown at waypoint 6 and 6 fall into this category, which are common cues that pilots encounter while navigating. The $\mathrm{x}$ axis represents the prior probability and the $\mathrm{y}$ axis represents the posterior probability, i.e., updated belief after seeing a visual cue. Agent A's prior and posterior probabilities are the same when applying Bayes' rule. Thus, the blue line with a slope of 1 indicates agent A's posterior probability is a function of prior probability, i.e., $y=x$. When subjects confuse evidence $p(d \mid H)$ with inference $p(H \mid d)$, then the posterior inferential probability becomes constant $(=p(d \mid H))$ regardless of the prior $p(H)$. This is misperception type 1 and is shown as a red dashed line in Fig. 5. As shown in Fig. 5(a), B1's posterior inference is constant $(p(d \mid H)=.95)$, and the misperception is mostly higher than the reasonably induced probability. Fig. 5(b) shows misperception type 2 (i.e., $p(d \mid H)$ $+p(d \mid \sim H)=1$ ) which is even more biased than misperception type 1; this misperception is always higher than A's perception. Posterior perceptions of all three agents (A, B1 and B2) are compared in Fig. 5(c). For example, when the prior probability was $=.7$, the posterior probability of A remained the same whereas B1 increased to .95 and B2 to .978 . This simulation shows that the suggested modeling framework can quantify human visual misperception compared to a Bayesian agent. Fig. 5(d) examines a case when $p(d \mid H)>p(d \mid \sim H)$, which can happen in a real situation. This is when the visual cue shown $d$ is more likely cue of $H$ than $\sim H$, e.g., $p(d \mid H)=.95, p(d \mid H)$ $p(d \mid \sim H)=.3$. In this case, agent A's posterior probability is slightly increased as shown in a black line compared to the straight 45 degree line of A's. Agent B's perceptions are the same as in the previous paragraph. Even with more likely cues given to pilots, misperceived posterior probabilities (both B1 and B2) are higher than a reasonably induced posterior.
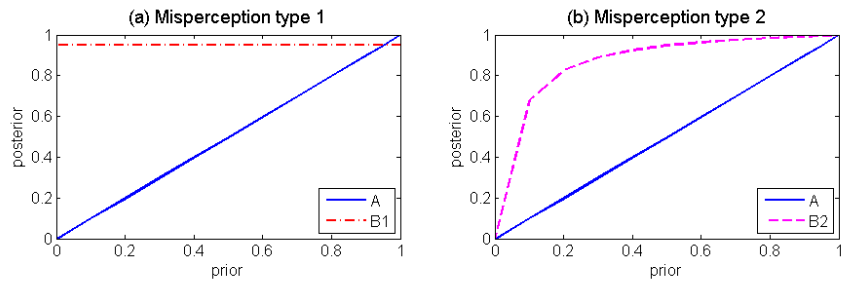

(c) Misperception type 182
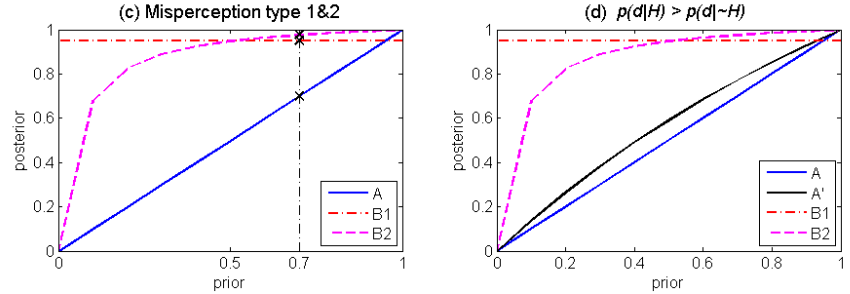

Figure 5. Differences in posterior inferential probabilities when the prior changes from 0 to 1 . A: Bayesian agent, $\mathrm{A}^{\prime}$ : Bayesian agent with more likely visul cue, B1: Misperception type 1, and B2: Misperception type 2 
Different visual cues can also result in variance in posteriors. In Fig. 5, the visual cue fidelity $p(d \mid H)$ was set to .95. Fig. 6(a) shows simulation results when given low, medium, and high fidelity cues. The visual cue fidelity $p(d \mid H)$ was defined $.3, .5$, and .9 respectively and shown in red dashed lines. As described earlier, misperception type 1 is a constant value depending on the cue fidelity. Thus, it seems pilots tend to favor the prior when given high fidelity cues whereas they reject the cue (biased sampling) when given low fidelity cues. (Table I summarized misperception type 1 occurs when $p(d \mid H)$ is high and type 3 when $p(d \mid H)$ is low.) In the case of misperception type 2, pilots favor prior probabilities more than agent A whenever $p(d \mid H)>0.5$. Fig. 6(b) simulation results shows when $p(d \mid H)$ is .6, .8, and .95.

\section{B. Case 2: waypoint 5 8, mispercetion progress modeling}

Fig. 3 showed a result trajectory of visual misperception which occurred in the overland navigation task. We can model the subject's misperception progress using the Bayesian misperception modeling framework. Case 1 demonstrated misperception modeling in a single point whereas this case (Case 2) exhibits dynamic evolution of a series of misperceptions in agent $\mathrm{Bs}$ and gaps between a correct agent $\mathrm{A}$.
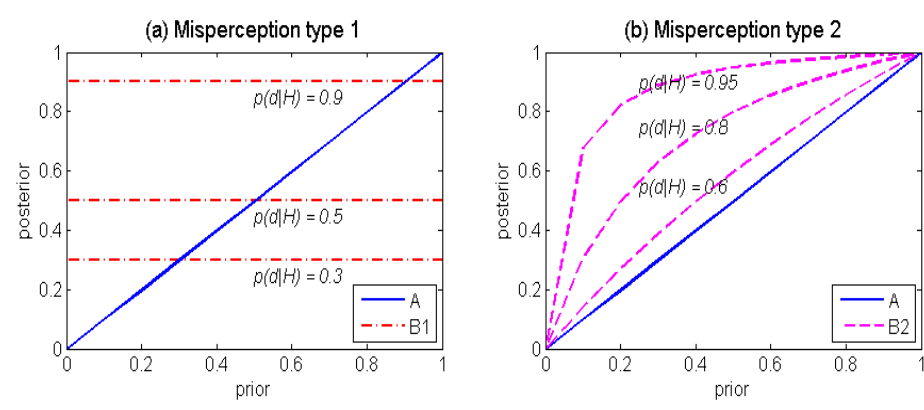

Figure 6. Differences in posterior inferential probabilities with different data fidelity $p(d \mid H)$. A: Bayesian agent, B1: Misperception type 1, and B2: Misperception type 2

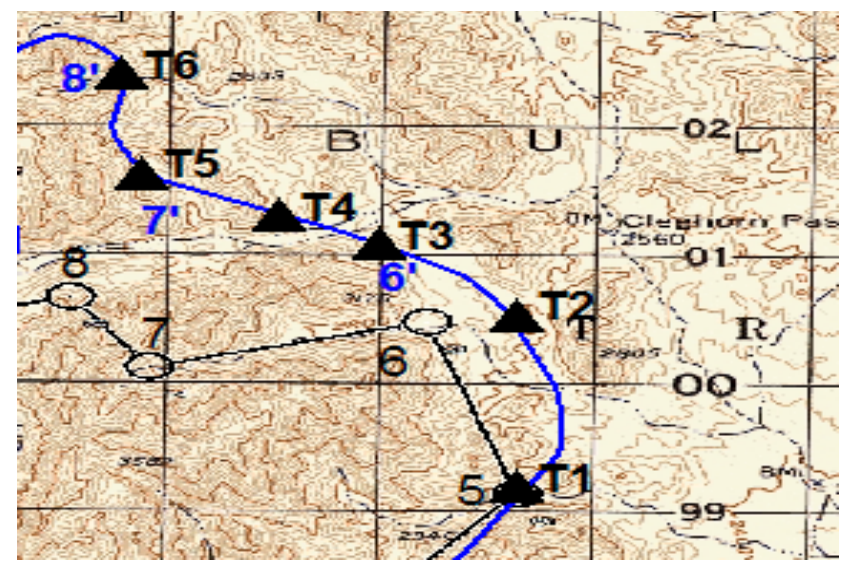

Figure 7. Subject 5's key perceptual situations T1 T6 along the actual flight trajectory.
Reviewing the flight session of subject 5 with the FEST (Flight and Eye Scan visualization Tool) developed with this study $[11,12]$, we were able to locate the following key situations which can affect perception update as shown in Fig. 7: $\mathrm{T} 1=$ initial position at waypoint $5, \mathrm{~T} 2=$ vicinity of true waypoint $6, \mathrm{~T} 3=$ waypoint $6^{\prime}$ with a valley shown in OTW view, T4 $=$ another valley shown in OTW view, T5 = waypoint 7', and T6 = waypoint 8'. Fig. 7 shows the key points along with the actual flight trajectory introduced in Fig. 3.

This dynamic evolution of Bayesian perception and misperception along the flight trajectory is shown in Fig. 8. The $\mathrm{x}$-axis represents key situations, i.e., discrete time events, and the $y$-axis is the updated inferential probability after passing the key points. At $\mathrm{T} 1$, subject 5 was actually at waypoint 5 believing he was on-track. Thus, we can suppose prior $p(H)$ is relatively high, e.g., .8. $H$ defines whether the pilot is on-track, thus, $p(H)$ is high when human perception and actual situation agrees. For example, $p(H) \sim .5$ indicates that navigation confidence is very low and $\mathrm{p}(\mathrm{H}) \sim .1$ indicates that the subject thinks he is off-track. At T2, the valley on waypoint 6 was not in OTW view, and the subject did not realize it. We can model all agents' posterior probabilities the same up to T2 as shown in Fig. 8. Up to this point, there exists no gap between agents A and Bs. At T3, the valley on the left is in sight in OTW view, and agent $\mathrm{A}, \mathrm{B} 1$, and $\mathrm{B} 2$ update their probabilities accordingly with a cue $p(d \mid H)=p(d \mid \sim H)=.95$. As shown in the previous section, agent A's probability remains the same whereas those of B1 and B2 increase to .95 and .987. At T4, a valley on the right was in sight, however, the pilot did not process this cue internally, which was a misperception type 3 (biasedsampling.) Thus, agent B1's and B2's perception remains the same applying misperception type 3 (B1 and B2 becomes $\mathrm{B} 1 \& \mathrm{~B} 3$ and $\mathrm{B} 2 \& \mathrm{~B} 3$ respectively), whereas agent A's perception is updated with $p(d \mid H)=.1$. The $p(d \mid H)$ is set to low because the valley on the right is not a possible visual cue if on-track. Thus, agent A's posterior at T4 decreased to .308 by applying Bayes' rule. The perception gaps between agents $\mathrm{A}$ and $\mathrm{Bs}$ are almost .7, suggesting agent $\mathrm{A}$ at least doubts its position or thinks it is off-track whereas agent B strongly believes it is on-track. At T5 and T6, visual cues similar to T2 are in sight and each agent updates its probability accordingly. The combination of three different misperception types aggravated the gap between agent A and B's perceptions.

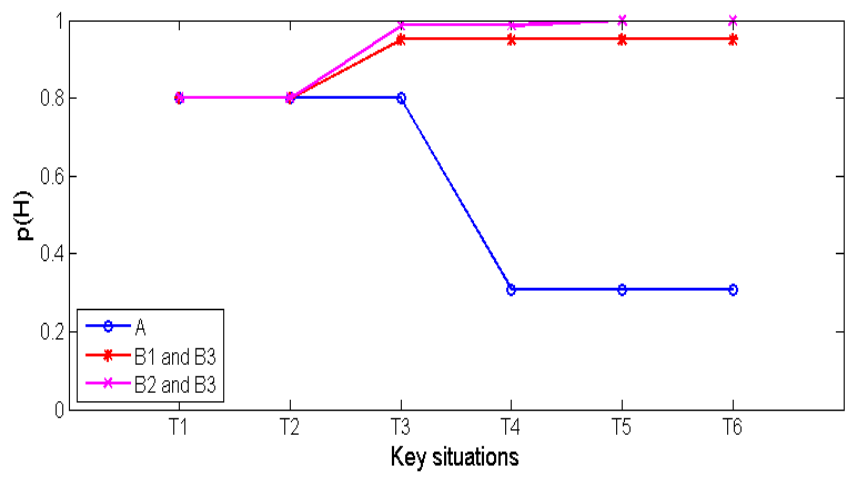

Figure 8. Dynamic evolution of perecptions. A: Bayesian agent, B1: Misperception type 1, B2: Misperception type 2, and and B3: Misperception type 3. T1 T6: key situations. 


\section{CONCLUSION AND FUTURE WORK}

We provided a formal modeling method to simulate visual misperception in overland navigation using Bayesian framework. Numerical simulation results based on experimental observation showed that our suggested modeling method can quantify visual misperception and compare with a Bayesian agent. This modeling technique can help explain errors found in an empirical investigation and be applied toward developing training navigation systems.

Our next research goal is to validate our model through another set of experiments in the same simulated environment. Developing training navigation systems and applying Brunswikian theory in the whole system will follow after that. We expect this misperception modeling technique to extend to any visual stimuli-based tasks such as terrain association, gesture recognition, etc.

We want to augment our model by including map view to capture recovery strategies of pilots. In one of the experts' example, the subject's debrief comments indicated that when faced with unexpected terrain features in view, his alternative possible location was based on map scan in the vicinity of recently-covered terrain. Although the subject was mistaken in the assumption that he was off course, he could not have mismatched terrain in view with a plausible alternative location if he had not been comparing terrain covered against multiple possible locations on the map. Without this robust hypothesis generation and testing, including terrain recently covered, it would not have been possible for the subject to identify the point at which he thought he had made the turn early. Of the expert navigators in this study, two made minor errors and corrected themselves, and each described similar structures for recovery in debrief comments. Modeling this recovery strategy in our model will help improve visual perception in overland navigation.

As promising as the results appear, some of the aspects in our study can be improved in further research. First, integrating a vision recognition algorithm on the OTW view to identify series of visual cues available to pilots will help develop a human visual misperception detection system. We used FEST to review visual cues along the flight trajectory in our study. However, if we can automate this process using the aforementioned vision recognition algorithm, that will be useful for implementing our visual misperception modeling framework in field settings. Second, we need to investigate how pilots interpret cues with medium fidelity. Our misperception modeling simulation results agree with experimental observations with high and low fidelity cues. However, we did not study misperceptions occurring when given medium fidelity cues. Third, dynamic evolution of visual misperception can be more formally established as a Bayesian Network introduced as applied in [14].

\section{ACKNOWLEDGMENT}

All authors are very much thankful to Prof. Ron Fricker for critical and helpful advice on the statistical analysis. We are grateful to Noah Loyd-Edelman and Jesse Huston for helping us in calibrating the experimental device.

\section{REFERENCES}

[1] A. Kirlik, “ Adaptive Perspective on Human-Technology Interaction: Methods and Models for Cognitive Engineering and Human-Computer Interaction", Oxford University Press, 2006

[2] T. Griffiths, M. Kalish, and S. Lewandowsky, "Theoretical and empirical evidence for the impact of inductive biases on cultural evolution," Philosophical Transactions of the Royal Society B: Biological Sciences, vol. 363, pp. 3503 3514, 2008.

[3] M.D. Lee, "Three case studies in the Bayesian analysis of cognitive models," Psychonomic Bulletin \& Review, vol. 15 (1), pp. 1 15, 2008

[4] R. Rensink, "Change detection," Annual Review of Psychology, vol. 53, pp. $245-277,2002$.

[5] R. Rensink, J. O'Regan, and J. Clark, "To see or not to see: The need for attention to perceive changes in scenes," Psychological Science, vol. 15(1), pp. 27-32. 1997.

[6] A. Mack and L. Rock, Inattentional Blindness, MIT Press, Cambridge, MA, 1997.

[7] D. Simons and C. Chabris, "Gorillas in our midst: Sustained inattentional blindness for dynamic events," Perception, vol. 28(9), pp. 1059-1074, 1999

[8] L. Stanov and J. Lee, "Confidence and cognitive test performance," Journal of Educational Psychology, vol. 100(4), pp.961-976, 2008.

[9] M. Fotta, M. Byrne, and M. Luther, "Developing a human error modeling architecture (HEMA)," In Proceedings of Human-Computer International 2005. Mahwah, NJ: Erlbaum.

[10] J. Anderson, D. Bothell, M. Byrne, S. Douglass, C. Lebiere, and Y. Qin, "An Integrated Theory of the Mind," Psychological Review, vol.111 (4), pp. 1036 1060, October 2004

[11] J. Sullivan, J. Yang, M. Day, and Q. Kennedy, "Training Simulation for Helicopter Navigation by Characterizing Visual Scan Patterns," Aviation, Space, and Environmental Medicine, accepted on June 2011.

[12] J. Yang, Q. Kennedy, J. Sullivan, and M. Day, "Understanding cognitive processes of helicopter navigation by characterizing visual scan patterns," in Proceedings of the $1^{\text {st }}$ Conference on Pioneering Convergence Technologies, Jeju Island, Republic of Korea, February 14 16, 2011.

[13] J. Sullivan, "Assessing neurophysiologic markers for training and simulation to develop expertise in complex cognitive tasks," Naval Postgraduate School, Ph.D. dissertation, Modeling Virtual Environment and Simulation (MOVES), Naval Postgraduate School, Monterey, CA, 2010 .

[14] J. Yang, Z.-H. Mao, L. Tijerina, T. Pilutti, J. Coughlin, and E. Feron, "Detection of driver fatigue caused by sleep deprivation," in IEEE Transactions on Systems, Man, and Cybernetics Part A: Systems and Humans, vol. 39 (4), pp. 694 705, 2009. 